\title{
CPU86017, a berberine derivative, attenuates cardiac failure through normalizing calcium le- akage and downregulated phospholamban and exerting antioxidant activity
}

\author{
Min-you QI ${ }^{\#}$, Yu FENG ${ }^{\#}$, De-zai DAI ${ }^{\star}$, Na LI, Yu-si CHENG, Yin DAI \\ Research Division of Pharmacology, China Pharmaceutical University, Nanjing 210009, China
}

\begin{abstract}
Aim: To investigate whether CPU86017, a berberine derivative, attenuates heart failure by blocking calcium influx and exerting its antioxidant activity.

Methods: Myocardial infarction was induced in male Sprague-Dawley rats for $17 \mathrm{~d}$ followed by isoproterenol (ISO) (5 mg/kg, sc) treatment for $5 \mathrm{~d}$ to reduce cardiac function. The rats were divided into 5 groups: sham operation, myocardial infarction (MI), MI plus ISO, and co-treated (in mg/kg, po) with either propranolol (PRO, 10) or CPU86017 (80). Hemodynamic measurements were conducted, and measurements of the redox system, calcium handling proteins and endothelin (ET) system in vivo were done. Furthermore, calcium flux studies and PLB immunocytochemistry were conducted in vitro.

Results: Compared to sham operation, $\mathrm{HF}$ was evident following $\mathrm{MI}$ and further worsened by ISO treatment. This occurred in parallel with downregulated mRNA and protein production of SERCA2a, PLB, and FKBP12.6, and was associated with upregulation of preproET-1, endothelin converting enzyme, and PKA mRNA production in the myocardium in vivo. Calcium leakage was induced by ISO treatment of isolated beating myocytes in vitro. These changes were attenuated by treatment with either PRO or CPU86017. PLB fluorescence in myocytes was downregulated by ISO treatment, and was relieved significantly by treatment with antioxidant aminoguanidine, ascorbic acid or CPU86017 in vitro.

Conclusion: HF, calcium leakage, downregulated PLB, FKBP12.6, SERCA2a production, and upregulated PKA were caused by ISO treatment, and were abolished by CPU86017 treatment. The beneficial effects of CPU86017 are attributable to its antioxidant and calcium influx blocking effects.
\end{abstract}

Keywords: berberine; FKBP12.6; SERCA2a; heart failure; calcium leakage; endothelin; oxidative stress; phospholamban; SpragueDawley rats

Acta Pharmacologica Sinica (2010) 31: 165-174; doi: 10.1038/aps.2009.180

\section{Introduction}

Heart failure (HF) is a progressive clinical syndrome with high mortality. Despite significant advances in understanding the mechanisms underlying this disease, current treatments for HF have not been satisfied. Calcium homeostasis is essential for maintaining normal cardiac performance, and relies on the balance of calcium influx via $L$-type calcium channels with calcium release via the activity of the ryanodine receptor type 2 (RyR2) in the sarcoplasmic reticulum ${ }^{[1]}$. RyR2 responds to small influxes of calcium, and triggers a relatively large

\footnotetext{
\# The two authors contributed equally to this work.

* To whom correspondence should be addressed.

E-mail dezaidai@vip.sina.com

Received 2009-07-29 Accepted 2009-11-16
}

amount of calcium to be released into the cytosol, thereby eliciting calcium excitation coupling for contractility ${ }^{[2]}$. The $\mathrm{Ca}^{2+}$ releasing channel is tightly closed in diastole due to modulation by the protein calstabin 2 (FKBP12.6), which associates with RyR ${ }^{[3]}$. The process of calcium homeostasis, however, is critically impaired due to hyperphosphorylation of protein kinase A (PKA) $)^{[4]}$. PKA hyperphosphorylated exists in a hyperadrenergic state ${ }^{[5]}$ elicited by isoproterenol (ISO) treatment, resulting in both arrhythmogenesis and $\mathrm{HF}^{[1]}$. Impaired calcium homeostasis may not be due solely to the effects of the RyR2-FKBP12.6 complex and the impact of hyperphosphorylation of PKA. Calcium homeostasis in the cardiac cycle requires appropriate activity of a pair of $\mathrm{Ca}^{2+}$ modulating proteins: SERCA2a (sarcoplasmic reticulum $\mathrm{Ca}^{2+}$ ATPase), and its regulator PLB (phospholamban). These proteins are respon- 
sible for swiftly pumping an excess of calcium back into the calcium store of the sarcoplasmic reticulum during diastole. Emerging data have shown that SERCA2 $\mathrm{a}^{[6]}$ and $\mathrm{PLB}^{[7]}$ may be actively involved in the pathogenesis of HF. In addition to PKA, hyperphosphorylated PKC $\varepsilon$ may also play an important role in this process ${ }^{[8,9]}$.

A growing body of evidence has shown that dysfunction of calcium handling proteins is mainly due to the abnormal formation of the RyR2-FKBP12.6 complex ${ }^{[1]}$, and may also be due to the downregulation of SERCA2a and PLB, as demonstrated by the pivotal role of cardiac insufficiency in diabetic cardiomyopathy ${ }^{[10]}$ and arrhythmogenesis ${ }^{[11,12]}$. Downregulation of FKBP12.6 found in failing hearts facilitates $\mathrm{Ca}^{2+}$ leakage at diastole, leaving more $\mathrm{Ca}^{2+}$ behind, resulting in depletion of $\mathrm{Ca}^{2+}$ stores in the sarcoplasmic reticulum. This impairs calcium excitation-coupling in the cardiac cycle, and is the basic mechanism underlying the pathologies of failing hearts ${ }^{[12,13]}$. Under the hyperadrenergic state, SERCA2a and PLB expression could also contribute to excess cytosolic $\mathrm{Ca}^{2+}$ at diastole. However, the relationship of PLB with HF has not been fully evaluated, and has only been described in a limited number of studies $^{[14,15]}$.

CPU86017 is derived from berberine and contains chemical modifications that result in an improvement in water solubility, calcium channel blocking activity, and antioxidative activity $^{[16]}$. We have shown that CPU86017 is effective in relieving pulmonary hypertension induced by either hypoxia ${ }^{[17]}$ or monocrotaline ${ }^{[18]}$. Interestingly, a recent study demonstrated a novel clinical use of the drug verapamil to prolong survival of patients suffering from CPVT (catecholaminergic polymorphism ventricular tachycardia). CPVT is due to a mutation in RyR2 ${ }^{[19]}$, which leads to defective association with FKBP12.6. CPVT is triggered by physical exercise, and is related to leakiness of RyR2 channels and defective/downregulated FKBP12.6 $6^{[20]}$. This condition can also be produced by ISO treatment ${ }^{[5]}$. This is likely due to an activated endothelin-1 (ET-1) system and oxidative stress ${ }^{[8]}$. As a result, abnormal FKBP12.6 can be ameliorated significantly by the endothelin receptor antagonists darusentan, a selective ETA (endothelin receptor A) blocker, or CPU0213, a dual ETA/B blocker ${ }^{[7]}$. Myocardium infarction (MI) stimulated by ISO administration to rats showed an increased tendency for exacerbated VF relative to MI alone, and this phenomenon was associated with a more depressed expression of RyR2 and SERCA2a in the left ventricle. Expression was recovered by CPU86017 treatment ${ }^{[6]}$. These results indicate that excessive ISO treatment produces a state of hyperadrenergic activity and impairs a family of calcium-modulating proteins. This disturbs $\mathrm{Ca}^{2+}$ homeostasis during the cardiac cycle, in which oxidative stress and an abnormal ET pathway are implicated. Oxidative stress plays a role in the downregulation of FKBP12.6, increasing the vulnerability for developing HF and arrhythmogenesis ${ }^{[21,22]}$. However, it is unclear whether these changes are causal factors to exacerbate HF by causing calcium leakage.

Here, we hypothesize that an infarcted rat heart stimulated by ISO could exaggerate cardiac dysfunction in parallel with reduced function of calcium handling proteins and the redox system in vivo, and that calcium leakage caused by ISO administration correlates with downregulation of PLB in vitro. Furthermore, we propose that these abnormalities could be recovered by CPU86017 treatment, mainly due to its antioxidative and calcium antagonist activity. In the present study, we determined the ability of CPU86017 to attenuate these abnormalities at the molecular level with implications towards HF in vivo and in vitro.

\section{Materials and methods Animals}

Our study conforms to the Guide for the Care and Use of Laboratory Animals published by the US National Institutes of Health (NIH Publication No 85-23, revised 1996). Male Sprague-Dawley rats (250-280 g) were used for in vivo experiments, and neonatal rats were used to isolate cardiomyocytes for in vitro experiments.

\section{Experimental protocol}

Rats were randomly divided into 5 groups: sham operation (Sham), myocardial infarction (MI) by coronary artery ligation, MI plus injection of ISO, and MI+ISO treated with either propranolol (PRO), or CPU86017. Rats were subjected to left coronary ligation, and were allowed to recover from the operation $^{[6]}$. HF developed chronically following MI for $17 \mathrm{~d}$. Treatment with ISO $(5 \mathrm{mg} / \mathrm{kg}$, sc) was continued for $5 \mathrm{~d}$ to exacerbate congestive cardiac failure. From the 18th day, cotreatment with either propranolol $(10 \mathrm{mg} / \mathrm{kg}$, po) or CPU86017 $(80 \mathrm{mg} / \mathrm{kg}, p o)$ was continued for $5 \mathrm{~d}$, whereas rats in the sham, MI, and MI+ISO groups received the same volume of normal saline.

\section{Hemodynamics}

Rats were anesthetized with urethane $(1.5 \mathrm{~g} / \mathrm{kg}$, ip), and a left ventricular cannulation was performed via the left common carotid, according to previously described methods ${ }^{[10]}$. The intracardiac pressure recordings for the left ventricular systolic pressure (LVSP), left ventricular end-diastolic pressure (LVEDP), maximum velocity of left ventricular systole $\left(\mathrm{LV}+\mathrm{d} p / \mathrm{d} t_{\max }\right)$, and maximum velocity of left ventricular diastolic pressure $\left(\mathrm{LV}-\mathrm{d} p / \mathrm{d} t_{\max }\right)$ were measured by a real-time hemodynamic analyzer (MPA2000, Shanghai Secondary Military Medical University ${ }^{[10]}$.

\section{Heart weight index}

Weights of the whole heart (HW), the left ventricle (LVW) and right ventricle (RVW) were measured and used to calculate the ratio to body weight (HW/BW, LVW/BW, and RVW/ $\mathrm{BW})$, respectively, and compared among the groups ${ }^{[10]}$.

\section{RT-PCR}

The mRNA levels for SERCA2a, PLB, RyR2, FKBP12.6, ECE (endothelin converting enzyme), preproET-1 (preproendothelin-1), and PKA were measured by semi-quantitative RT-PCR ${ }^{[10]}$. Briefly, RNA was extracted from cardiac tissue 
by TRIzol, and was reverse transcribed into cDNA by MMLV RT with a profile of $10 \mathrm{~min}$ at $25^{\circ} \mathrm{C}, 30 \mathrm{~min}$ at $48^{\circ} \mathrm{C}$ and 2 min at $95^{\circ} \mathrm{C}$. One microgram of cDNA was used for PCR in a $25 \mu \mathrm{L}$ final reaction volume. PCR products were stained with ethidium bromide and visualized under UV light (GDS8000, Sygene, England). The density of each band was measured using professional image analysis software. The ratio of the mRNA of the gene of interest against an internal standard GAPDH oxidase was calculated. The nucleotide sequences of primers chosen are listed in Table 1.

Table 1. Oligonucleotide primers used for reverse transcript-polymerase chain reactions.

\section{Oligonucleotide primers}

\begin{tabular}{ll}
\hline SERCA2a & Sense: 5'-CCGTATCCGATGACAATG-3' \\
& Antisense: 5'-CCAGGCTCCAGGTAGTT-3' \\
PLB & Sense: 5'-TACCTTACTCGCTCGGCTATC-3' \\
& Antisense: 5'-CAGAAGCATCACAATGATGCAG-3' \\
RyR2 & Sence: 5'-GAATCAGTGAGTTACTGGGCATGG-3' \\
& Antisense: 5'-CTGGTCTCTGAGTTCTCCAAAAGC-3' \\
FKBP12.6 & Sense: 5'-GTGAAGGCAGGAAGGAA-3' \\
& Antisense: 5'-GCAGCCAACAGAAGATAAG-3' \\
ECE & Sense: 5'-GGACTTCTTCAGCTACGCCTGT-3' \\
& Antisense: 5'-CTAGTTTCGTTCATACACGCACG-3' \\
PreproET-1 & Sense: 5'-AGCAATAGCATCAAGGCATC-3' \\
& Antisense: 5'-TCAGACACGAACACTCCCTA-3' \\
PKA & Sense: 5'-CAAGAGAGTCAAGGGCAGGAC-3' \\
& Antisense: 5'-CAACCTTTCTCGGTAAATCGC-3' \\
GAPDH & Sense: 5'-GCTGGGGCTCACCTGAAGG-3' \\
& Antisense: 5'-GGATGACCTTGCCCACAGCC-3' \\
\hline
\end{tabular}

\section{Western blot}

Quantitative analysis of SERCA2a, PLB, and FKBP12.6 protein levels was performed as previously described ${ }^{[10]}$. Briefly, a portion of cardiac tissue was homogenized in 4 volumes of extraction buffer and centrifuged at $10000 \times g$ for $10 \mathrm{~min}$ at $4{ }^{\circ} \mathrm{C}$. After determining the protein concentration, supernatants were stored at $-20{ }^{\circ} \mathrm{C}$ before use. The samples were heated at $95^{\circ} \mathrm{C}$ in loading buffer and fractionated by sodium dodecyl sulfate polyacrylamide gel electrophoresis (SDS-PAGE) on a $10 \%$ gel. Following transfer to a nitrocellulose membrane and blocking with nonfat milk ( $5 \% w / v)$, the blot was incubated with primary antibody overnight at $4{ }^{\circ} \mathrm{C}$. After washing, the blot was then incubated with horseradish peroxidase conjugated goat immunoglobulin (IgG) for $2 \mathrm{~h}$ at room temperature. Antigen was detected with a 3,3'-diaminobenzidine (DAB) kit, and visualized by an imaging acquisition instrument (Labwork, England) and quantified by densitometry. The ratio of tested protein against the sham was calculated quantitatively.

\section{Biochemistry assay}

A portion of cardiac mass was homogenized in normal saline, and the levels of nitric oxide (NO), malondialdehyde (MDA), superoxide dismutase (SOD), glutathione peroxidase (GSHpx) and the activities of induced NOS (iNOS) were determined following the instructions of the reagent kits (Nanjing Jiancheng Bioengineering Institute, China $)^{[10]}$.

\section{Calcium transients}

Single cardiac myocytes were enzymatically isolated from whole ventricles of adult rats as described before with some modifications ${ }^{[23]}$. In brief, male rats $(250 \pm 50 \mathrm{~g})$, were heparinized and anaesthetized with ethylurethane $(1.5 \mathrm{~g} / \mathrm{kg}$, ip). The heart was quickly excised and retrogradely perfused through the aortic cannula mounted on a Langendorff apparatus at $37{ }^{\circ} \mathrm{C}$ with a $\mathrm{Ca}^{2+}$-free Tyrode solution for about $5 \mathrm{~min}$. This was then switched to the same solution containing 0.33 $\mathrm{mg} / \mathrm{mL}$ type II collagenase (Worthington, USA) for $12 \pm 2 \mathrm{~min}$. The ventricles were dissected and cardiac myocytes were collected and dispersed and stored in a high- $\mathrm{K}^{+}$, low $-\mathrm{Cl}^{-} \mathrm{Kraft}-$ Brühe (KB) solution.

Measurements of cytosolic $\left[\mathrm{Ca}^{2+}\right]_{i}$ were performed as described previously ${ }^{[23,24]}$, with some modifications. Briefly, cardiomyocytes loaded with Fluo-3 were transferred to a 300$\mu \mathrm{L}$ perfusion chamber, placed on an inverted microscope (Olympus IX71, Japan), perfused with compounds of interest or vehicle, and gassed with $95 \% \mathrm{O}_{2}+5 \% \mathrm{CO}_{2}$ at room temperature. Cyclic contractions of cardiomyocyte were paced by square wave pulses of $40 \mathrm{~V}$, with a $5-\mathrm{ms}$ duration at $0.5 \mathrm{~Hz}$ in a field of electric stimulation. Imaging of Fluo-3 fluorescence within cardiac cycles was recorded 5 min after perfusion of tested compounds or vehicle, and the signals were converted into absolute $\left[\mathrm{Ca}^{2+}\right]_{\mathrm{i}}$ by the calibration formula, $\left[\mathrm{Ca}^{2+}\right]_{\mathrm{i}}=K_{\mathrm{d}}{ }^{*}(F-$ $\left.F_{\min }\right) /\left(F_{\max }-F\right)$. Here, $K_{\mathrm{d}}$ represents the dissociation constant for $\mathrm{Ca}^{2+}$-bound Fluo-3, $F$ was obtained from $F=F_{\text {cell }}-F_{\text {bak }}$, where $F_{\text {cell }}$ is the mean fluorescent intensity measured in myocytes, and $F_{\text {bak }}$ is the fluorescent intensity of the background. $F_{\max }$ represented the maximum fluorescence of cells permeable to $\mathrm{Ca}^{2+}$, and was obtained by adding $5 \mathrm{mmol} / \mathrm{L} \mathrm{A23187} \mathrm{to}$ Tyrode's solution containing $10 \mathrm{mmol} / \mathrm{L} \mathrm{Ca}^{2+}$. $F_{\text {min }}$ was calculated from $F_{\min }=1.25^{*} F_{\mathrm{Mn}}-0.25^{*} F_{\max }$, where $F_{\mathrm{Mn}}$ was obtained by adding $5 \mathrm{mmol} / \mathrm{L} \mathrm{MnCl}_{2}$ to $5 \mathrm{mmol} / \mathrm{L} \mathrm{A23187-treated} \mathrm{myo-}$ cytes $^{[24]}$.

\section{Solutions Normal}

Tyrode's solution contained (in mmol/L): $135 \mathrm{NaCl}, 5.4 \mathrm{KCl}$, $1.8 \mathrm{CaCl}_{2}, 2 \mathrm{MgSO}_{4}, 0.33 \mathrm{NaH}_{2} \mathrm{PO}_{4}, 10$ glucose and 10 HEPES ( $\mathrm{pH}$ adjusted to 7.4 with $\mathrm{NaOH}$ ). The $\mathrm{Ca}^{2+}$-free Tyrode's solution used for the cell isolation procedure was prepared by simply omitting $\mathrm{CaCl}_{2}$ from the normal Tyrode's solution. The $\mathrm{KB}$ solution for cell preparation contained (in mmol/L): $40 \mathrm{KCl}$, $3 \mathrm{MgCl}_{2}, 20 \mathrm{KH}_{2} \mathrm{PO}_{4}, 50$ L-glutamic acid, 20 taurine, $70 \mathrm{KOH}$, 10 glucose, 0.5 EGTA and 10 HEPES ( $\mathrm{pH}$ adjusted to 7.3 with $\mathrm{KOH})$. All solutions used during the procedure were oxygenated and maintained at $37{ }^{\circ} \mathrm{C}$. ISO $(100 \mathrm{nmol} / \mathrm{L})$ was directly added to normal Tyrode's solution. Propranolol at $10^{-6}$ $\mathrm{mol} / \mathrm{L}$ or CPU86017 at $10^{-7}, 10^{-6}$, and $10^{-5} \mathrm{~mol} / \mathrm{L}$ was coincubated with ISO. Peak (systolic), variant (difference between systolic and diastolic) and trough (diastolic) concentrations of 
$\left[\mathrm{Ca}^{2+}\right]_{\mathrm{i}}$ were calculated.

\section{Immunocytochemistry of phospholamban}

The procedure was conducted as previously described ${ }^{[7]}$. Briefly, ventricular myocytes were isolated from adult rats, plated onto laminin-coated slip chambers, and cultured at $37^{\circ} \mathrm{C}$ in MEM/EBSS serum-free medium. Four hours later, test drugs were added to chambers for 8 groups: (1) Control; (2) ISO $\left(10^{-6} \mathrm{~mol} / \mathrm{L}\right)$; (3 and 4) ISO+aminoguanidine (Ami, $10^{-5}$ or $\left.10^{-6} \mathrm{~mol} / \mathrm{L}\right)$, a blocker for iNOS, with potent antioxidant activity; (5 and 6) ISO+ascorbic acid $\left(\mathrm{VC}, 10^{-4}\right.$ or $\left.10^{-5}\right)$, a typical antioxidant; and (7 and 8) ISO+CPU86017 $\left(10^{-5}\right.$ or $\left.10^{-6}\right)$.

\section{Immunocytochemistry assay for $\mathrm{PLB}^{[7]}$}

Briefly, $24 \mathrm{~h}$ after drug incubation, the ventricular myocytes were fixed using $4 \%$ paraformaldehyde for $15 \mathrm{~min}$ at $37^{\circ} \mathrm{C}$ and were rinsed and then incubated for $30 \mathrm{~min}$ with $2 \%$ bovine serum albumin in PBS to reduce nonspecific binding. After overnight incubation at $5{ }^{\circ} \mathrm{C}$ with the primary antibody, cells were rinsed and incubated with the secondary antibody conjugated with fluorescent isothiocyanate (FITC), for $2 \mathrm{~h}$ at room temperature. Stained cells were imaged by fluorescence and were photographed. The fluorescence image was converted into gray density in the accumulated data to be compared among groups.

\section{Statistics}

All the data are expressed as mean \pm SD. The differences between groups are compared with ANOVA, and $P<0.05$ is considered statistically significant.

\section{Results}

\section{Cardiac dysfunction}

Cardiac function was depressed following myocardial infarction, and was further worsened by injection of ISO. Compared to the sham group, the systolic and diastolic functions were compromised significantly in the ISO group, with declines in LVSP by $-30.2 \%(P<0.01), \mathrm{LV}+\mathrm{d} p / \mathrm{d} t_{\max }$ by $-46.7 \%(P<0.01)$, and $\mathrm{LV}-\mathrm{d} p / \mathrm{d} t_{\max }$ by $-53.3 \%(P<0.01)$. LVEDP was elevated by $+786.8 \%(P<0.01)$, relative to normal (Figure 1A-1D). An impairment in cardiac function was demonstrated by MI, and was further compromised by ISO administration $(P<0.05)$, compared to MI alone. Medication with either propranolol or CPU86017 for $5 \mathrm{~d}$ significantly reversed cardiac dysfunction, where CPU86017 preserved LVSP by $66.5 \%(P<0.01)$, LVEDP by $90.5 \%(P<0.01), \mathrm{LV}+\mathrm{d} p / \mathrm{d} t_{\max }$ by $83.7 \%(P<0.01)$, and $\mathrm{LV}-\mathrm{d} p / \mathrm{d} t_{\max }$ by $84.7 \%(P<0.01)$ relative to the ISO group.

\section{Cardiac hypertrophy}

Myocardium hypertrophy was found following MI and ISO medication, demonstrating an increase in the heart weight
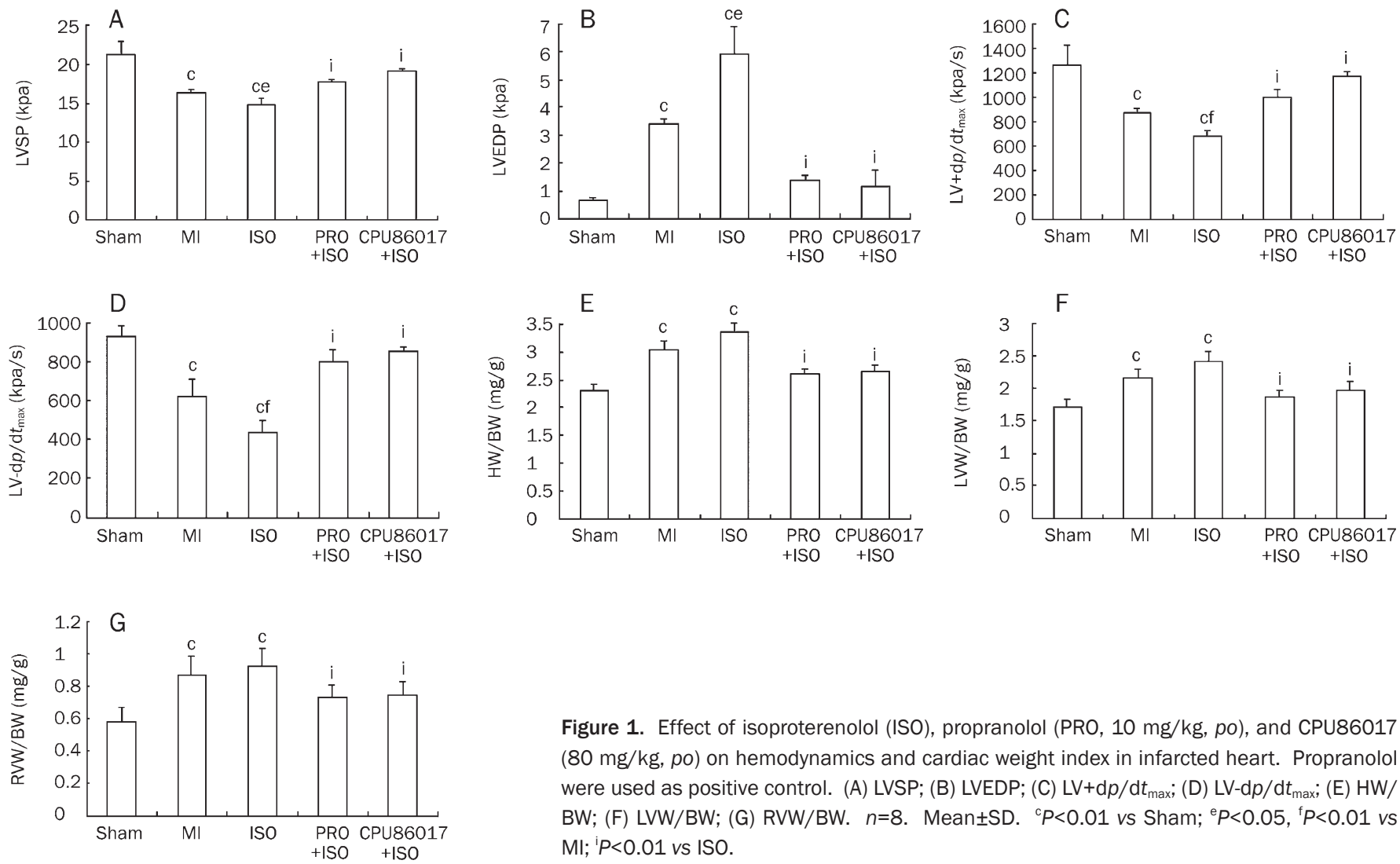

Figure 1. Effect of isoproterenolol (ISO), propranolol (PRO, $10 \mathrm{mg} / \mathrm{kg}$, po), and CPU86017 $(80 \mathrm{mg} / \mathrm{kg}, \mathrm{po})$ on hemodynamics and cardiac weight index in infarcted heart. Propranolol were used as positive control. (A) LVSP; (B) LVEDP; (C) LV+dp/dt $t_{\max }$; (D) LV-dp/dt $t_{\max } ;$ (E) HW/ BW; (F) LVW/BW; (G) RVW/BW. $n=8$. Mean \pm SD. ${ }^{\mathrm{c}} P<0.01$ vs Sham; ${ }^{\mathrm{e}} P<0.05,{ }^{\mathrm{f}} P<0.01$ vs $\mathrm{MI}$; $P<0.01$ vs ISO. 
indexes $\mathrm{HW} / \mathrm{BW}$, LVW/BW, and RVW/BW by $46.1 \%, 41.3 \%$ and $60.3 \%(P<0.01)$ respectively (Figure $1 \mathrm{E}-1 \mathrm{G})$. Both propranolol and CPU86017 were able to significantly reverse hypertrophy.

\section{Abnormal calcium handling molecules}

To characterize the beginning of cardiac dysfunction initiated by MI and worsened in the presence of ISO, we focused our attention on measuring the changes in the expression of SERCA2a and PLB. Downregulation of the two molecules was significant in the MI group, and further downregulation was achieved by ISO treatment $(P<0.05$, Figure 2$)$. In the MI heart, mRNA levels of SERCA2a (Figure 2A) and PLB (Figure 2C) in the left ventricle decreased, in agreement with a reduction in the respective protein levels. These were found to be reduced by $48.8 \%$ and $56.7 \%$, respectively, (both $P<0.01$ ), relative to the sham operation group (Figure 2B, 2D). Further reduction in mRNA and protein levels $(P<0.05)$, was seen in the MI+ISO group, compared to MI alone with exception of the SERCA2a protein. Both propranolol and CPU86017 were effective to the same extent in abolishing the abnormalities.

Expression of RyR2 and FKBP12.6 in the myocardium was
A
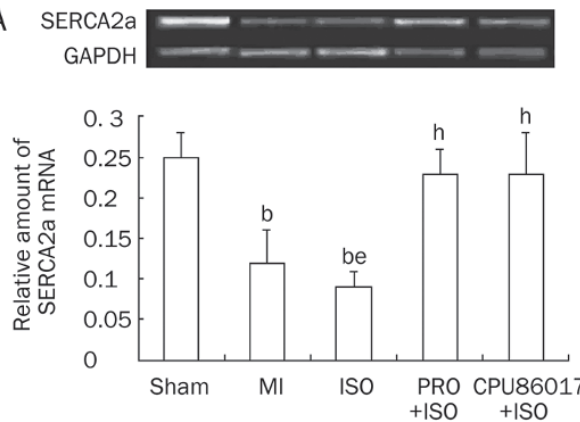

C
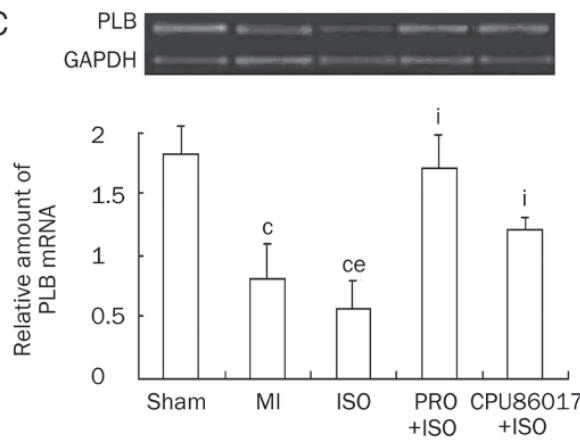

\section{$\mathrm{E}$}
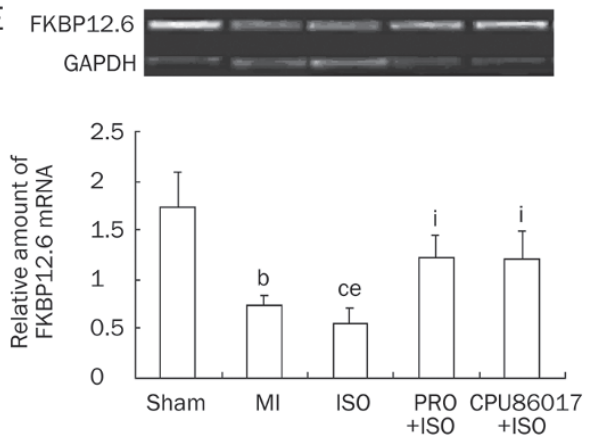

G
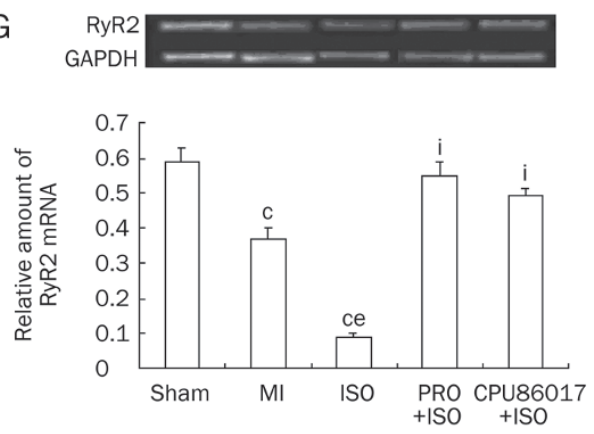

B
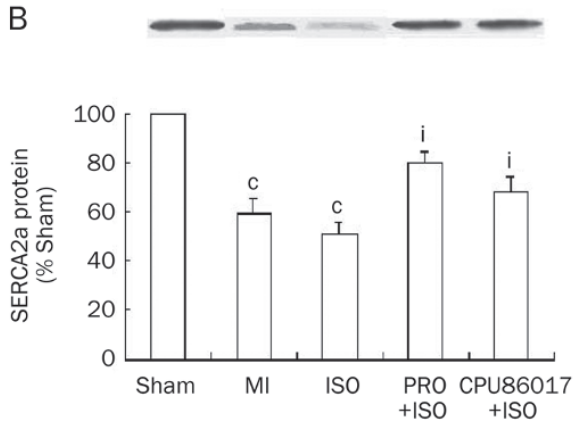

D

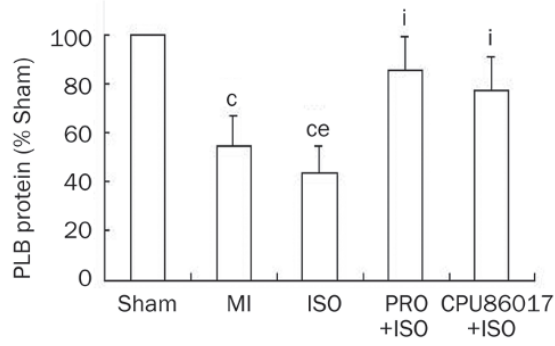

$\mathrm{F}$

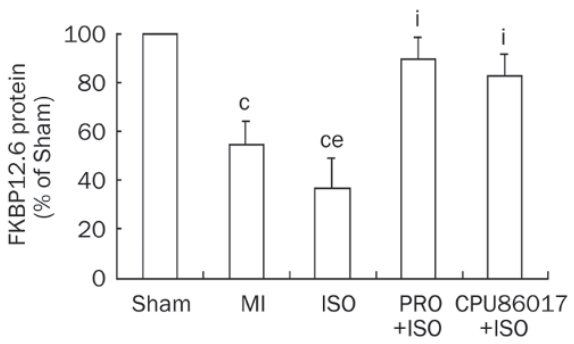

Figure 2. Effect of isoproterenolol (ISO), propranolol (PRO), and CPU86017 on expression of calcium handing proteins in myocardial infarction. (A) SERCA2a mRNA; (B) SERCA2a protein; (C) PLB mRNA; (D) PLB protein; (E) FKBP12.6 mRNA; (F) FKBP12.6 protein; (G) RyR2 mRNA. $n=4$. Mean \pm SD. ${ }^{\mathrm{b}} P<0.05,{ }^{\mathrm{c}} P<0.01$ vs Sham; ${ }^{\mathrm{e}} P<0.05$ vs Ml; ${ }^{\mathrm{h}} P<0.05$, i $P<0.01$ vs ISO. 
depressed in response to MI combined with ISO administration. Expression of these proteins was significantly depressed in response to $\mathrm{MI}(P<0.05)$ relative to the sham, and worsened by ISO $(P<0.05)$, as compared to MI alone (Figure 2E, $2 \mathrm{G}$ ). Levels of the FKBP12.6 protein were decreased in parallel with the changes in cardiac dysfunction $(P<0.01)$ (Figure $2 F)$. Intervention with propranolol and CPU86017 was successful in significant reversal of the abnormalities.

\section{Calcium transient activity and calcium leakage}

Calcium transient studies in rat ventricular myocytes were carried out to show cyclic changes in traces of Fluo-3 in regular beating cardiomyocytes elicited by electrical field stimulation. The peak, variance (difference between peak and endof-diastolic value), and trough values at diastole were calculated. After application of $100 \mathrm{nmol} / \mathrm{L}$ isoproterenol, calcium transients were significantly augmented in the three values. An increment in diastolic levels over the normal level was recognized as calcium leakage. Changes in $\left[\mathrm{Ca}^{2+}\right]_{i}$ induced by ISO were respectively reversed by either treatment with propranolol at $1 \mu \mathrm{mol} / \mathrm{L}$ or CPU86017 at $10 \mu \mathrm{mol} / \mathrm{L}$. CPU86017 was mild in inhibiting these changes, and no effect was found at $0.1 \mu \mathrm{mol} / \mathrm{L}$ (Figure 3).

\section{Upregulation of ECE, preproET-1 and PKA mRNA}

We were next interested in investigating whether cardiac dysfunction in MI followed by activation of the $\beta$-adrenoceptor by ISO medication was related to an activated ET system in the myocardium. Upregulation of the myocardial ET system was reflected by escalating mRNA levels of ECE and preproET-1 (Figure 4A, 4B) in the infarcted left ventricle $(P<0.01)$ compared to the sham. A further increase in pp-ET-1 was caused by ISO administration, relative to MI rats $(P<0.05)$. Treatment with propranolol and CPU86017 remarkably decreased these changes $(P<0.01)$.

PKA is considered an important downstream effector, subsequent to $\beta$-adrenoceptor activation. Expression of PKA mRNA appeared to be upregulated significantly $(P<0.01)$ in MI, and further escalation in PKA mRNA by ISO was substantial, in parallel to changes in cardiac function $(P<0.05)$ (Figure 4C). PKA upregulation was markedly inhibited by either propranolol or CPU86017 ( $P<0.01$, Figure 4).

\section{Oxidative stress}

Oxidative stress was evident in the myocardium found in both the MI alone group and the combined ISO injection groups, with an elevation of MDA content by 107\% $(P<0.01)$,
A

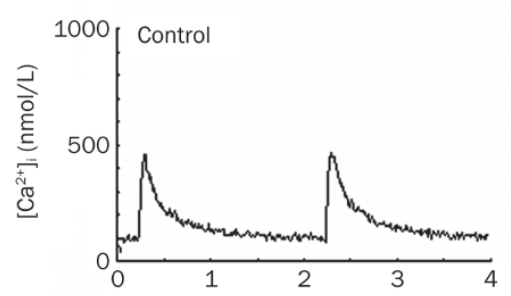

D

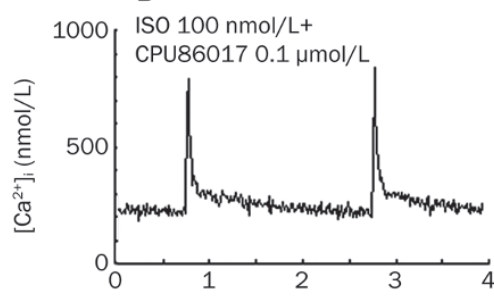

B

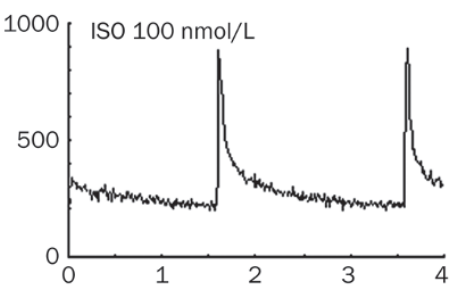

E

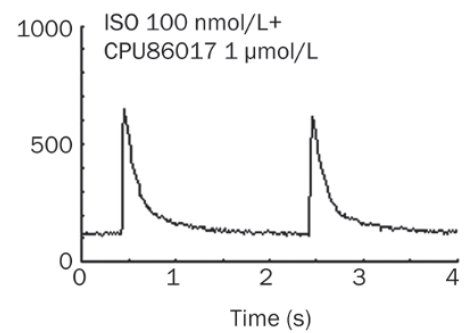

C

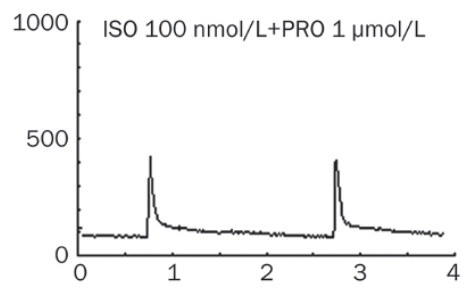

$\mathrm{F}$

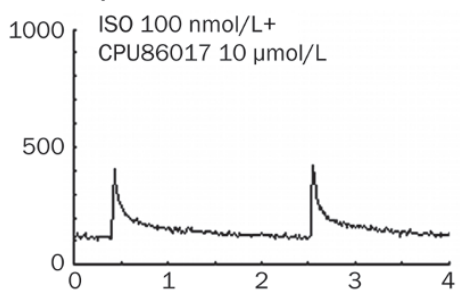

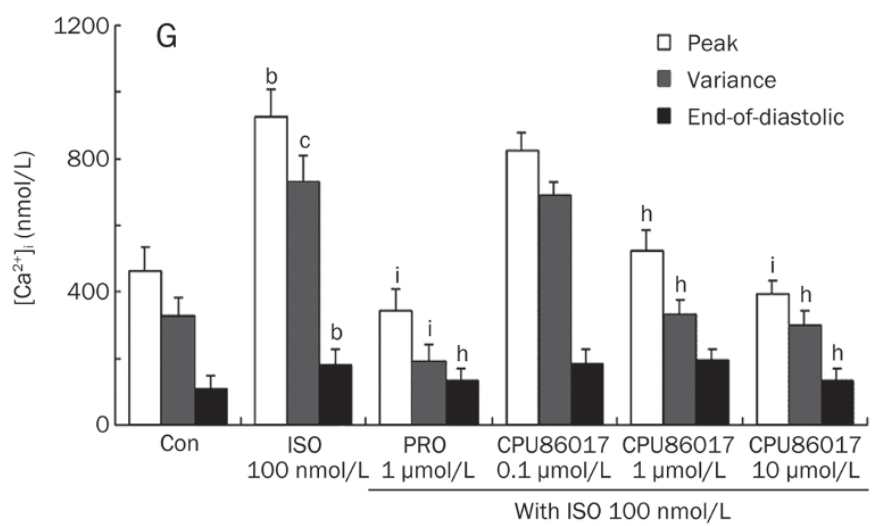

Figure 3. Effect of CPU86017 (0.1, 1.0, and $10 \mu \mathrm{mol} / \mathrm{L})$ and propranolol (PRO, $1 \mu \mathrm{mol} / \mathrm{L}$ ) on calcium transients in beating primary myocytes paced by field electric stimulation. (A) Control; (B) Incubated with ISO (100 nmol/L); (C) ISO plus PRO (1 $\mu \mathrm{mol} / \mathrm{L})$; (D) ISO plus CPU86017 (0.1 $\mu \mathrm{mol} / \mathrm{L})$; (E) ISO plus CPU86017 (1 $\mu \mathrm{mol} / \mathrm{L}) ;(\mathrm{F})$ ISO plus CPU86017 (10 $\mu \mathrm{mol} / \mathrm{L}) ;(\mathrm{G})$ Cumulated data of peak (at systole), variant (difference between the peak and trough) and trough (at diastole) were compared among groups. $n=8-10$. Mean \pm SD. ${ }^{\mathrm{b}} P<0.05,{ }^{\mathrm{c}} P<0.01$ vs Control; ${ }^{\mathrm{h}} P<0.05$, ${ }^{i} P<0.01$ vs ISO. 
A
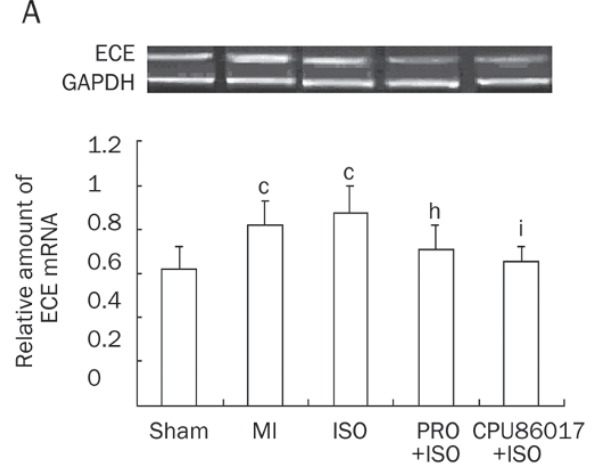

B
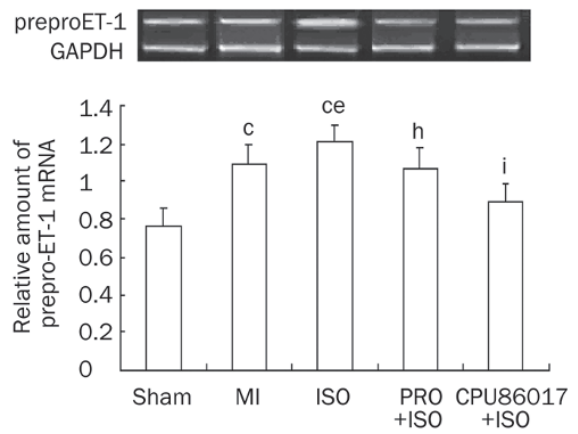

C
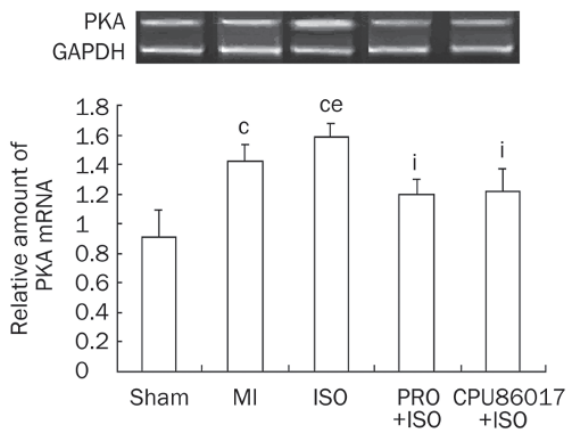

Figure 4. Effect of isoproterenolol (ISO), propranolol (PRO), and CPU86017 on up-regulation of mRNA expression of ECE (A), prepro-ET-1 (B), and PKA (C) in the failing heart caused by infarction. $n=4$. Mean \pm SD. ${ }^{\mathrm{C}} P<0.01$ vs Sham; ${ }^{\mathrm{e}} P<0.05$ vs $\mathrm{Ml} ;{ }^{\mathrm{h}} P<0.05,{ }^{\mathrm{i}} P<0.01$ vs ISO.

and decreased SOD and GSH-px content by $40.3 \%$ and $48.2 \%$ $(P<0.01)$, respectively, in the ISO group relative to the sham group. A further significant decline in SOD activity was observed following ISO medication (Figure 5A-5C).

Activity of iNOS and NO content: Cytokines are frequently involved in failing hearts, and the activity of iNOS was measured, showing enhanced iNOS activity following the MI procedure $(P<0.01)$ relative to the sham group. An excess of NO content was the product of activated iNOS, and served as an oxidant, contributing adversely to cardiac function and further elevating NO levels $(P<0.05)$ during ISO administration, compared to the MI group. These changes play a large role in oxidative stress, and were attenuated by either propranolol or CPU86017 treatment (Figure 5D, 5E).

\section{Immunocytochemistry of phospholamban}

Phospholamban (PLB) is a critical modulator of SERCA2a, and modulates the uptake process of $\mathrm{Ca}^{2+}$ into the $\mathrm{Ca}^{2+}$ store of the sarcoplasmic reticulum. We found that PLB was downregulated by ISO medication, as seen by a loss of fluorescence in these treated myocytes relative to normal myocytes (Figure 6). Aminoguanidine, a specific inhibitor of NNOS $^{[25]}$ was sufficient for recovering the fluorescence image of PLB protein, as was the antioxidant ascorbic acid ${ }^{[26]}$ (but at higher concentrations). Recovery of fluorescence of PLB was also achieved by an application of CPU86017 to levels comparable to those of antioxidants (Figure 6).

\section{Discussion}

There are four major findings emanating from the present study: 1) Compromised cardiac function and hypertrophy were caused by MI and worsened by ISO medication in vivo; 2) Changes in cardiac function by MI combined with ISO stimulation occurred in parallel with changes in FKBP12.6, SER-
A
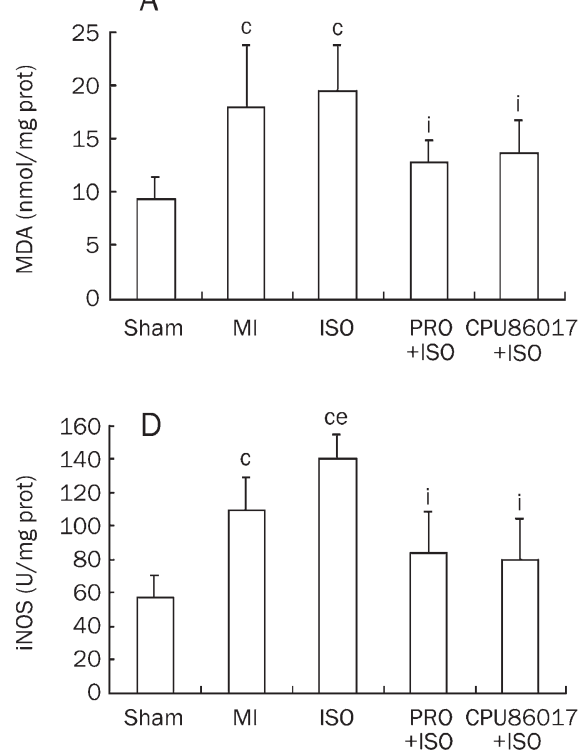

B
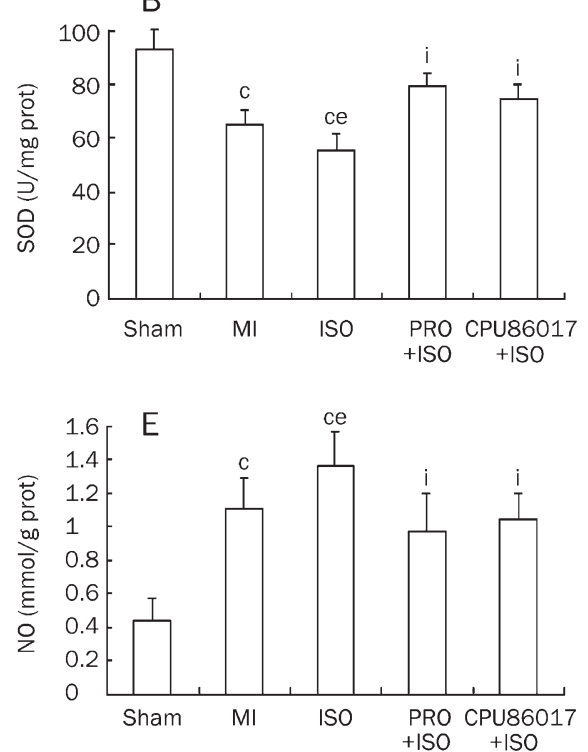

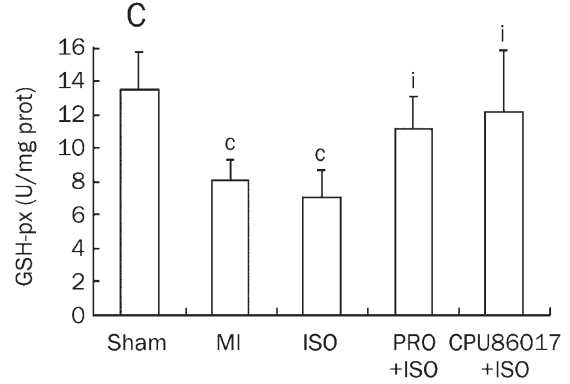

Figure 5. Effect of isoproterenolol (ISO), propranolol (PRO), and CPU86017 on the redox system in the myocardium caused by infarction. (A) MDA; (B) SOD activity; (C) GSH-px; (D) iNOS activity; and (E) NO content. $n=8$. Mean \pm SD. ${ }^{\circ} P<0.01$ vs Sham; ${ }^{e} P<0.05$ vs MI; $P<0.01$ vs ISO. 
A
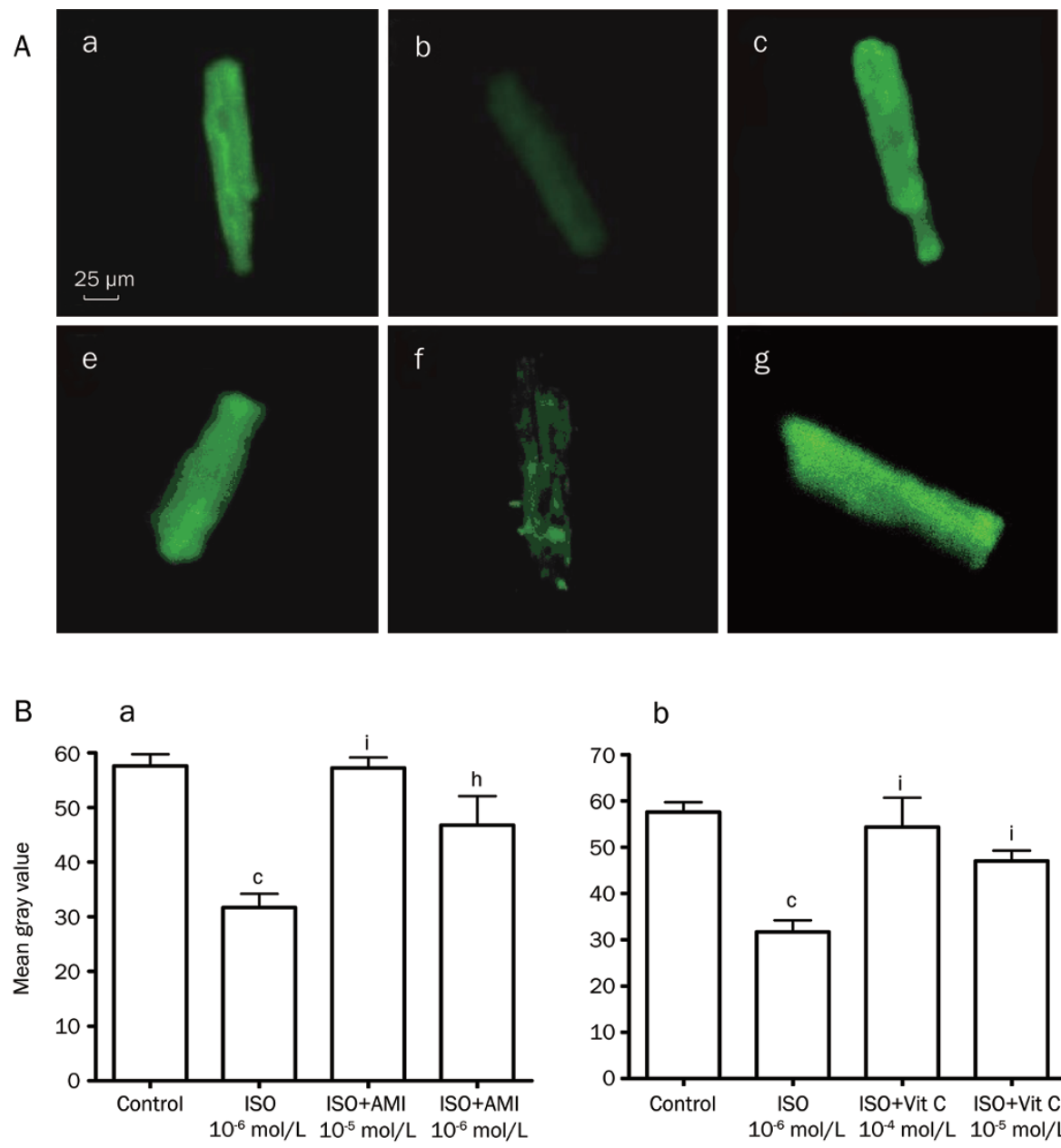

b

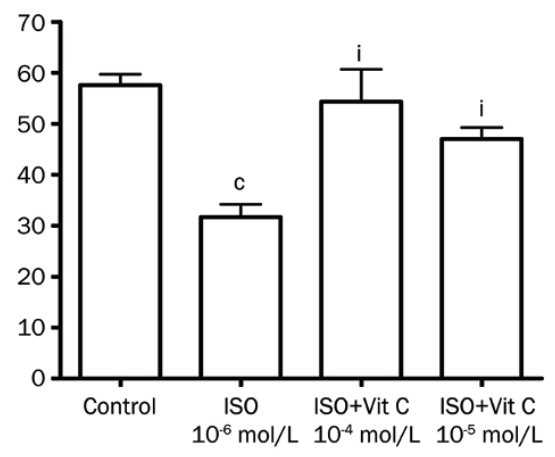

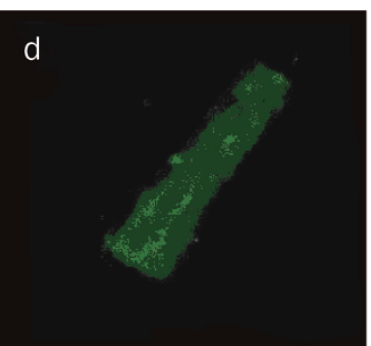

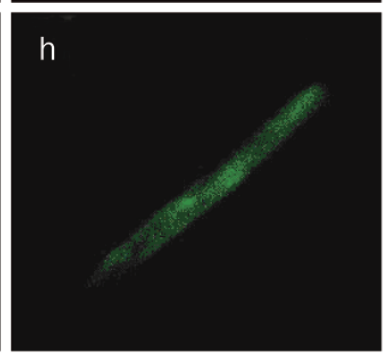

C

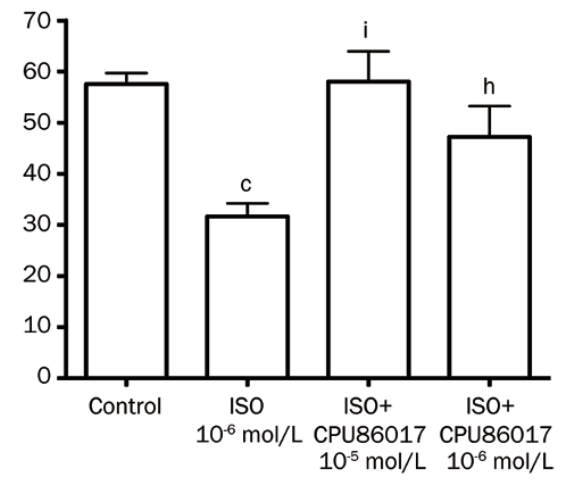

Figure 6. Effect of antioxidants aminoguanidine (AMI), ascorbic acid (Vit C), and CPU86017 on downregulation of phospholamban (PLB) in the primary rat myocytes induced by isoproterenolol (ISO). (A) Fluorescent image: a, Control; b, ISO $1 \mu \mathrm{mol} / \mathrm{L} ; \mathrm{c}$, ISO+AMI $10 \mu \mathrm{mol} / \mathrm{L}$; d, ISO+AMI $1 \mu \mathrm{mol} / \mathrm{L}$; e,

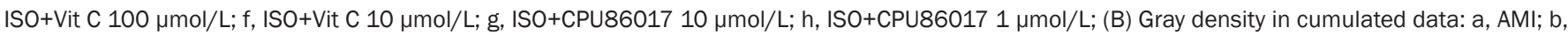
Vit C; c, CPU86017. $n=8$. Mean \pm SD. ${ }^{\mathrm{c}} P<0.01$ vs Control; ${ }^{\mathrm{h}} P<0.05,{ }^{\mathrm{i}} P<0.01$ vs ISO.

CA2a, PLB, RyR2, and PKA levels, and overt oxidative stress in the myocardium; 3) $\mathrm{Ca}^{2+}$ leakage was elicited in the presence of ISO, and responded to CPU86017 treatment in vitro; 4) Calcium leakage caused by ISO is related to the downregulation of PLB due to the activity of oxidants, and can be reversed by CPU86017 treatment.

With regards to the treatment of $\mathrm{HF}$, more attention should be paid to the biomarkers of calcium homeostasis in the cardiac cycle ${ }^{[27]}$. The elevated $\mathrm{Ca}^{2+}$ levels at the end of diastole that result from calcium leakage are major factors in the pathogenesis of failing hearts. This leakage is due to an unstable and defective RyR2 caused by the dissociation of FKBP12.6 $6^{[1,8]}$. In addition, other factors, including downregulation of SERCA2a and PLB expression, are of interest because of their contribution to calcium leakage by less efficient $\mathrm{Ca}^{2+}$ uptake into the pool of the sarcoplasmic reticulum. Defective SERCA2a disturbs intracellular calcium regulation, adversely affecting excitation-contraction coupling in cardiac myocytes, and consequently impairs the systolic and diastolic functions of the heart ${ }^{[28]}$. Thus, exacerbated cardiac dysfunction by ISO medication may be the result of two factors: abnormal calcium releasing activity and abnormal calcium uptake behavior at the sarcoplasmic reticulum. Changes in cardiac function by the two steps coincided with changes in biomarkers including RyR2 and its modulator FKBP12.6, as well as SERCA2a and its modulator PLB. This is in line with other published findings on aggravating cardiac failure by ISO injection ${ }^{[5,29-31]}$.

Hyperphosphorylation of the calcium handling proteins, including those such as RyR2, in the hyperadrenergic state is mainly caused by PKA. However, calmodulin kinase II is also implicated in this process ${ }^{[32]}$. In addition, the effects of ISO treatment may be partly mediated by $\mathrm{PKC} \varepsilon$ phosphorylation ${ }^{[8]}$ to downregulate RyR2, FKBP12.6, SERCA2a, and PLB, with involvement of oxidative stress and related ET pathways ${ }^{[7]}$. $\mathrm{Ca}^{2+}$ released into the cytosol requires an equal amount of $\mathrm{Ca}^{2+}$ uptake in the sarcoplasmic reticulum during diastole, and is essential in maintaining $\mathrm{Ca}^{2+}$ homeostasis and normal relaxation $^{[33]}$. An excess of ROS mediates downregulation of 
FKBP12.6, and the antioxidative activity of tocopherol sufficiently mitigates ISO-induced downregulation of calcium handling proteins as well as hyperphosphorylation of $P K C \varepsilon^{[8]}$. Thus, an attenuation of the hyperadrenergic state induced by downregulation of FKBP12.6 by CPU86017 is possibly the consequence of its antioxidative activity.

SERCA2a activity positively correlates with cardiac performance, and downregulation of SERCA2a is frequently associated with downregulation of $\mathrm{PLB}^{[34]}$. A clinically applicable, high-efficiency, percutaneous means of therapeutic gene delivery with adenovirus expressing a pseudophosphorylated mutant AdS16E recovers the phosphorylation of PLB, resulting in an improvement of HF in large animals ${ }^{[35]}$. With downregulated SERCA2a and PLB, the speed and amount of calcium uptake into the sarcoplasmic reticulum are reduced, rendering high diastolic $\mathrm{Ca}^{2+}$ levels at diastole. This is critical to developing cardiac insufficiency. Downregulated PLB as seen by immunohistochemistry after ISO treatment correlates with cardiac dysfunction. We showed that CPU86017 was sufficient to mitigate these changes to the same extent as other antioxidants such as ascorbic acid and aminoguanidine (a specific blocker for iNOS), and consequently reduces the damage to the myocardium by attenuating the genesis of $\mathrm{NO}$ and possibly the potent oxidant ONOO- in the presence of ISO.

CPU86017, derived from berberine ${ }^{[16]}$, reduced ISO elevated diastolic $\mathrm{Ca}^{2+}$ in a concentration-dependent way that was at least partially attributed to its antioxidant activity. This resembled the activity of ascorbic acid in the present study and that of tocopherol ${ }^{[7]}$. In contrast, PKA phosphorylation by ISO medication is markedly suppressed by a blockade on endothelin receptors ${ }^{[7,8]}$ and an activated ET system typically found in pulmonary artery hypertension is sufficiently suppressed by CPU86017 ${ }^{[17,18]}$. The antioxidant activity of CPU86017 provides relief to arrhythmogenesis in thyroxininduced cardiomyopathy in rats ${ }^{[36]}$ and reduces calcium leakage in isolated myocytes from thyroxin-induced arrhythmic rat hearts ${ }^{[37]}$. These results indicate that CPU86017 initiates beneficial effects on both HF and cardiac dysrhythmia related to normalized PLB expression, likely stemming from its antioxidative properties. Propanolol used in conjunction with verapamil, a classic calcium antagonist, has been proven to be more effective in prolonging the survival of patients with mutated RyR2 presenting CPVT ${ }^{[38]}$ than propranolol alone. Thus, a restriction of calcium influx by the calcium antagonism of CPU86017 contributes in part to attenuating ISO-induced $\mathrm{HF}$ and arrhythmogenesis. Furthermore, calcium leakage can be blocked by intervention with compound JTV519 ${ }^{[39]}$, which shares a similar profile of multi-channel blocking activity including an inhibitory effect on $\mathrm{Ca}^{2+}$ influx with CPU86017 ${ }^{[40]}$. Furthermore, an antioxidative activity is effective in ameliorating abnormal PLB and calcium leakage during septic shock due to improved mitochondria activity ${ }^{[4]}$.

Limitations: Concerning activated PKA in the presence of $\beta$-adrenoceptor stimulation by ISO, we did not directly observe hyperphosphorylated PKA protein, but upregulated PKA mRNA only. However, following $\beta$-receptor activation in our previous reports we found that that besides phosphorylation of PKA, ISO induced hyperphosphorylation of PKC $\varepsilon$. This could be reproduced by $\mathrm{H}_{2} \mathrm{O}_{2}$, and is suppressed by propranolol, endothelin receptor antagonist, or the antioxidant tocopherol ${ }^{[8]}$. Thus, both PKA and PKC $\varepsilon$ are involved in ISO induced hyperphosphorylation and are responsible for downregulation of calcium handling proteins. Furthermore, this process is likely mediated by ROS.

In conclusion, cardiac dysfunction was deteriorated in two steps by MI and ISO stimulation in parallel with changes in downregulation of calcium handling proteins (PLB, SERCA2a, FKBP12.6, and RyR2) leading to calcium leakage. These abnormalities are likely to be related to the genesis of ROS. The antioxidative activity of CPU86017 contributes to relieving HF via normalizing downregulation of PLB and calcium leakage. CPU86017 therefore has the potential to treat stressrelated exacerbation of HF and arrhythmias in clinical practice.

\section{Acknowledgements}

This project was supported by the National Natural Foundation of China № 30670760 and the Major State Basic Research Development Program of the People's Republic of China (№ 2006CB503807).

\section{Author contribution}

Min-you QI and Yu FENG conducted the project and data processing. Na LI and Yu-si CHENG assisted with data processing and manuscript preparation. De-zai DAI and Yin DAI designed the research project by setting up hypotheses, raised suitable targets for investigation and revised the manuscript.

\section{References}

1 Wehrens XH, Lehnart SE, Reiken S, Vest JA, Wronska A, Marks AR. Ryanodine receptor/calcium release channel PKA phosphorylation: a critical mediator of heart failure progression. Proc Natl Acad Sci USA 2006; 103: 511-8.

2 Lehnart SE, Wehrens XH, Kushnir A, Marks AR. Cardiac ryanodine receptor function and regulation in heart disease. Ann NY Acad Sci 2004; 1015: 144-59.

3 Lehnart SE. Novel targets for treating heart and muscle disease: stabilizing ryanodine receptors and preventing intracellular calcium leak. Curr Opin Pharmacol 2007; 7: 225-32.

4 Reiken S, Gaburjakova M, Guatimosim S, Gomez AM, D'Armiento J, Burkhoff D, et al. Protein kinase A phosphorylation of the cardiac calcium release channel (ryanodine receptor) in normal and failing hearts. Role of phosphatases and response to isoproterenol. J Biol Chem 2003; 278: 444-53.

5 Ellison GM, Torella D, Karakikes I, Purushothaman S, Curcio A, Gasparri C, et al. Acute beta-adrenergic overload produces myocyte damage through calcium leakage from the ryanodine receptor 2 but spares cardiac stem cells. J Biol Chem 2007; 282: 11397-409.

6 Wang HL, Dai DZ, Gao E, Zhang YP, Lu F. Dispersion of ventricular mRNA of RyR2 and SERCA2 associated with arrhythmogenesis in rats. Acta Pharmacol Sin 2004; 25: 738-43.

7 Feng Y, Tang XY, Dai DZ, Dai Y. Reversal of isoproterenol induced downregulation of phospholamban and FKBP12.6 is mediated by antagonism of endothelin receptors of CPU0213. Acta Pharmacol 
Sin. 2007; 28: 1746-54.

8 Li N, Jia N, Dai DZ, Dai Y. Endothelin receptor antagonist CPU0213 and vitamin E reverse downregulation of FKBP12.6 and SERCA2a: a role of hyperphosphorylation of PKC epsilon. Eur J Pharmacol 2008; 591: 211-8.

9 Cheng YS, Dai DZ, Dai Y. Isoproterenol disperses distribution of NADPH oxidase, MMP-9, and pPKCepsilon in the heart, which are mitigated by endothelin receptor antagonist CPU0213. Acta Pharmacol Sin 2009; 30: 1099-106.

10 Qi MY, Xia HJ, Dai DZ, Dai Y. A novel endothelin receptor antagonist CPU0213 improves diabetic cardiac insufficiency attributed to upregulation of the expression of FKBP12.6, SERCA2a, and PLB in rats. J Cardiovasc Pharmacol 2006; 47: 729-35.

11 Phrommintikul A, Chattipakorn N. Roles of cardiac ryanodine receptor in heart failure and sudden cardiac death. Int J Cardiol 2006; 112: 142-52.

12 Marks AR. Novel therapy for heart failure and exercise-induced ventricular tachycardia based on 'fixing' the leak in ryanodine receptors. Novartis Found Symp 2006; 274: 132-47.

13 Gyorke S, Terentyev D. Modulation of ryanodine receptor by luminal calcium and accessory proteins in health and cardiac disease. Cardiovasc Res 2008; 77: 245-55.

14 Shattock MJ. Phospholemman: its role in normal cardiac physiology and potential as a druggable target in disease. Curr Opin Pharmacol 2009; 9: 160-6.

15 Gao MH, Tang T, Guo T, Miyanohara A, Yajima T, Pestonjamasp K, et al. Adenylyl cyclase type $\mathrm{VI}$ increases Akt activity and phospholamban phosphorylation in cardiac myocytes. J Biol Chem 2008; 283: 33527-35.

16 Dai DZ. CPU86017: a novel Class III antiarrhythmic agent with multiple actions at ion channels. Cardiovasc Drug Rev 2006; 24 : 101-15.

17 Zhang TT, Cui B, Dai DZ, Tang XY. Pharmacological efficacy of CPU 86017 on hypoxic pulmonary hypertension in rats: mediated by direct inhibition of calcium channels and antioxidant action, but indirect effects on the ET-1 pathway. J Cardiovasc Pharmacol 2005; 46: 727-34.

18 Zhang TT, Cui B, Dai DZ, Su W. CPU 86017, p-chlorobenzyltetrahyd roberberine chloride, attenuates monocrotaline-induced pulmonary hypertension by suppressing endothelin pathway. Acta Pharmacol Sin 2005; 26: 1309-16.

19 Sumitomo N, Harada K, Nagashima M, Yasuda T, Nakamura Y, Aragaki $\mathrm{Y}$, et al. Catecholaminergic polymorphic ventricular tachycardia: electrocardiographic characteristics and optimal therapeutic strategies to prevent sudden death. Heart 2003; 89: 66-70.

20 Kontula K, Laitinen PJ, Lehtonen A, Toivonen L, Viitasalo M, Swan $\mathrm{H}$. Catecholaminergic polymorphic ventricular tachycardia: recent mechanistic insights. Cardiovasc Res 2005; 67: 379-87.

21 Nakamura R, Egashira K, Machida Y, Hayashidani S, Takeya M, Utsumi $\mathrm{H}$, et al. Probucol attenuates left ventricular dysfunction and remodeling in tachycardia-induced heart failure: roles of oxidative stress and inflammation. Circulation 2002; 106: 362-7.

22 Brigadeau F, Gelé P, Marquié C, Soudan B, Lacroix D. Ventricular arrhythmias following exposure of failing hearts to oxidative stress in vitro. J Cardiovasc Electrophysiol 2005; 16: 629-36.

23 Kao JP, Harootunian AT, Tsien RY. Photochemically generated cytosolic calcium pulses and their detection by fluo-3. J Biol Chem 1989; 264 : 8179-84.

24 Huang ZJ, Dai DZ, Li N, Na T, Ji M, Dai Y. Calcium antagonist property of CPU228, a dofetilide derivative, contributes to its low incidence of torsades de pointes in rabbits. Clin Exp Pharmacol Physiol 2007; 34: 310-7.
25 Ishibashi Y, Takahashi N, Tokumaru A, Karino K, Sugamori T, Sakane T, et al. Activation of inducible NOS in peripheral vessels and outcomes in heart failure patients. J Card Fail 2008; 14: 724-31.

26 Han JM, Chang BJ, Li TZ, Choe NH, Quan FS, Jang BJ, et al. Protective effects of ascorbic acid against lead-induced apoptotic neurodegeneration in the developing rat hippocampus in vivo. Brain Res 2007; 1185: 68-74.

27 Minamisawa S, Sato Y, Cho MC. Calcium cycling proteins in heart failure, cardiomyopathy and arrhythmias. Exp Mol Med 2004; 36: 193-203.

28 Lokuta AJ, Maertz NA, Meethal SV, Potter KT, Kamp TJ, Valdivia HH, et al. Increased nitration of sarcoplasmic reticulum $\mathrm{Ca}^{2+}$-ATPase in human heart failure. Circulation 2005; 111: 988-95.

29 Du XJ, Cox HS, Dart AM, Esler MD. Sympathetic activation triggers ventricular arrhythmias in rat heart with chronic infarction and failure. Cardiovasc Res 1999; 43: 919-29.

30 Elhendy A, Sozzi FB, van Domburg RT, Bax JJ, Geleijnse ML, Roelandt JR. Relation among exercise-induced ventricular arrhythmias, myocardial ischemia, and viability late after acute myocardial infarction. Am J Cardiol 2000; 86: 723-9.

31 Priori SG, Napolitano C, Memmi M, Colombi B, Drago F, Gasparini M, et al. Clinical and molecular characterization of patients with catecholaminergic polymorphic ventricular tachycardia. Circulation 2002; 106: 69-74.

32 Currie S. Cardiac ryanodine receptor phosphorylation by CaM Kinase II: keeping the balance right. Front Biosci 2009; 14: 5134-56.

33 Chakraborti S, Das S, Kar P, Ghosh B, Samanta K, Kolley S, et al. Calcium signaling phenomena in heart diseases: a perspective. Mol Cell Biochem 2007; 298: 1-40.

34 Vittone L, Mundina-Weilenmann C, Mattiazzi A. Phospholamban phosphorylation by CaMKII under pathophysiological conditions. Front Biosci 2008; 13: 5988-6005.

35 Kaye DM, Preovolos A, Marshall T, Byrne M, Hoshijima M, Hajjar R, et al. Percutaneous cardiac recirculation-mediated gene transfer of an inhibitory phospholamban peptide reverses advanced heart failure in large animals. J Am Coll Cardiol 2007; 50: 253-60.

36 Hao JM, Dai DZ, Yu F. The exaggerated oxidative stress developed in cardiomyopathy by over-doses of thyroxin and these were reversed partly by CPU86017, a derivative of tetra-hydro-berberine. J Advan Pharm Sci 2005; 29: 417-21.

$37 \mathrm{Na} \mathrm{T}$, Huang ZJ, Dai DZ, Zhang Y, Dai Y. Abrupt changes in FKBP12.6 and SERCA2a expression contribute to sudden occurrence of ventricular fibrillation on reperfusion and are prevented by CPU86017. Acta Pharmacol Sin 2007; 28: 773-82.

38 Rosso R, Kalman JM, Rogowski O, Diamant S, Birger A, Biner S, et al. Calcium channel blockers and beta-blockers versus beta-blockers alone for preventing exercise-induced arrhythmias in catecholaminergic polymorphic ventricular tachycardia. Heart Rhythm 2007; 4: 1149-54.

39 Yano M, Kobayashi S, Kohno M, Doi M, Tokuhisa T, Okuda S, et al. FKBP12.6-mediated stabilization of calcium-release channel (ryanodine receptor) as a novel therapeutic strategy against heart failure. Circulation 2003; 107: 477-84.

40 Yamamoto T, Yano M, Xu X, Uchinoumi H, Tateishi H, Mochizuki M, et al. Identification of target domains of the cardiac ryanodine receptor to correct channel disorder in failing hearts. Circulation 2008; 117 : 762-72.

41 Hassoun SM, Marechal X, Montaigne D, Bouazza Y, Decoster B, Lancel $\mathrm{S}$, et al. Prevention of endotoxin-induced sarcoplasmic reticulum calcium leak improves mitochondrial and myocardial dysfunction. Crit Care Med 2008; 36: 2590-6. 